\title{
Questioning the mental health effects of community-based interventions: evaluation of a women empowerment programme
}

\author{
Juliane M Hoss', Linda E Blokland² \\ and Roland Weierstall',3
}

\begin{abstract}
This study conducted an empirical evaluation of a pre-existing women empowerment community programme purporting to address negative consequences of traumatic experiences and genderbased violence while facilitating posttraumatic growth. It was conducted in Tembisa, a South African township, with 103 women aged 18-56 $(M=27.77, S D=8.29)$. The women were assessed before the start of the programme. A 3-month follow-up assessed 70 women: 39 in the intervention group, $3 \mathrm{I}$ in the control group. Factors assessed included demographic information, traumatic experiences, depressive symptoms (Patient Health Questionnaire-9), and symptoms of posttraumatic stress disorder (PTSD Symptoms Scale-Interview for DSM-5). Results on the mental health measures indicated the general need for a programme supporting the women in coping with their challenges due to poverty, unemployment, and inequality in the community. Results suggested that the programme had little or even negative influence on the women's functionality and mental health symptoms. The strong focus on traumatic experiences of the women empowerment programme seems to be inadequate for the specific needs of this target group. This article argues against the implementation of interventions without tailoring them to the specific needs of the target populations. A collaboration between researchers and practitioners is recommended to adequately pursue the goals of such programmes.
\end{abstract}

\section{Keywords}

Gender-based violence, posttraumatic growth, poverty, structural violence, trauma, women empowerment programme

\footnotetext{
'University of Konstanz, Germany

2University of Pretoria, South Africa

3MSH Medical School Hamburg, Germany
}

Corresponding author:

Linda E Blokland, University of Pretoria, Private Bag X20, Hatfield, Pretoria 0028, South Africa.

Email: linda.blokland@gmail.com 
This study evaluates a pre-existing women's victim empowerment programme that was developed by a non-governmental organisation (NGO) for implementation through a local youth centre. The programme targets women who have been referred by local police stations and victim empowerment centres following traumatic experiences. The programme has been presented regularly in the past. The youth centre implementing the programme requested a structured evaluation aimed at analysing the suitability of the programme in addressing the needs in the target community of vulnerable women with a continued high risk for traumatisation. The purpose of the evaluation was to investigate possible improvements to the programme design.

The specific women's victim empowerment programme investigated in this study purports to impart empowerment through addressing women's experience of victimisation and traumatisation, with an emphasis on posttraumatic stress disorder (PTSD) and related symptoms such as depression. The rationale behind the design is based on the model of posttraumatic growth (PTG) (Tedeschi \& Calhoun, 1996). The programme was developed by the NGO to address the specific living conditions of women in systemically disadvantaged areas in South Africa and their experience of high levels of violence and traumatisation. It contains different intervention modules which consist of a blend of psychoeducation and person-centred group therapy using a narrative approach. The modules include a focus on self-enhancement, value clarification, building relationships, stress management and managing negative emotions, women's health, and gender-based violence and traumatisation.

In accordance, this study aimed at providing data on the relevance of the focus as well as the possible impact of the programme on the reduction of measures of depression as well as PTSD symptoms and level of functioning. The study focussed on the programme presentations between February and November 2016. These took place in Tembisa, a low socio-economical 'township' area close to Johannesburg.

Community-based interventions such as the one under study, delivered by NGOs, and designed to improve mental health, are widespread, and particularly located in resource-poor settings that often depend on external resources. In using pre- and post-test measurements, this study investigated the suitability of this programme design for the specific needs of the women in the target population.

In engaging in this programme review, the researchers acknowledge that there is a need for such programmes in South Africa where the majority of people live in communities with a lack of resources (Shefer et al., 2008). Adverse living factors existing in these areas include crime and violence (Pillay, 2008) and a high risk for victimisation. It is common to have experienced at least one or more traumatic events in a lifetime (Dinan, McCall, \& Gibson, 2004).

In conducting the study, it was acknowledged that existing research indicates high correlations between traumatic experiences and impaired mental health, especially symptoms of PTSD (Dinan et al., 2004). In addition, several studies have indicated that individuals, who develop PTSD, live with related symptoms such as depression and significant impairment over long periods of time (Lombardo \& Gray, 2005).

However, studies further indicate that traumatisation is linked with a broad variety of negative consequences, such as a lower quality of life, lower self-esteem, as well as some behavioural changes (Orsillo \& Batten, 2005). Also indicated is increased risk to experience worsening social relationships (Bisson, Brayne, Ochberg, \& Everly, 2007), a negative change in attachment styles, and a change in cognitions about the self, others, and the world (Fallot \& Harris, 2002). There may also be experiences of emotional numbness as well as difficulties regulating emotions (Fallot \& Harris, 2002) to the extent of becoming aggressive or increasing personal risk (Saltzman, Pynoos, Layne, Steinberg, \& Aisenberg, 2001). Based on the existing literature reviewed, it is suggested that trauma interventions may need to address a broad spectrum of challenges (Bisson et al., 2007). 
Considering these complex consequences is especially important in work with populations that suffer from multiple traumatic events and associated negative consequences (Orsillo \& Batten, 2005).

In accordance, the so-called 'second generation' treatments for PTSD go beyond targeting the symptoms that constitute a PTSD diagnosis (Lombardo \& Gray, 2005) and extend to consider contextual factors as well (Fallot \& Harris, 2002). A core goal of such a comprehensive strategy is to assist individuals to gain mastery over their personal experiences (Kerig, Fedorowicz, Brown, \& Warren, 2000) and build self-efficacy (Killen et al., 2008). These treatments of PTG (Tedeschi \& Calhoun, 1996) build on the unique capacities and resilience (Bonanno, 2004) of individuals instead of focusing solely on their negative experiences (Bisson et al., 2007).

It is particularly difficult to design adequate interventions for communities that are shaped by a lack of resources and high levels of traumatisation and violence. A number of factors impact treatment possibilities, such as economic restrictions and often inadequate structural conditions (Havenaar, Geerlings, Vivian, \& Collinson, 2008). The living conditions of a person influences psychopathological symptoms and often hinders the healing process after traumatic experiences (Harvey, 2007). A threat of further victimisation or traumatisation (Saltzman et al., 2001) may exist. Common misperceptions can hinder the work of practitioners, who may overestimate the frequency of PTSD symptoms and have difficulty identifying the actual needs of these communities. Individual (Berkowitz, Stover, \& Marans, 2011) as well as environmental (Murray, Nelson, Polan, Maticka-Tyndale, \& Ferris, 2004) factors influence the resilience of individuals. Several studies have indicated that, contrary to the expectations of many practitioners (Pupavac, 2001), few individuals develop PTSD while most recover to their previous level of functioning without any intervention (Bisson et al., 2007).

It is important to take contextual factors into consideration while offering affected individuals guidance to identify realistic opportunities of healing on a community level (Campbell \& MacPhail, 2002) and change within their specific living conditions (De Arellano, Deblinger, Cohen, Danielson, \& Mannarino, 2005). This study aimed to explore the impact on mental health of participants in a women's empowerment programme. The community intervention programme under study identified its focus as addressing negative consequences of traumatic experiences and gender-based violence. The impact of not tailoring the programme to the specific needs of the target population is considered.

\section{Method}

The study used a pre- and post-test measurement in a quasi-experimental design.

\section{Participants}

The participants were drawn from women already recruited between January and February 2016, into the programme run by the local youth centre. Women are referred to the programme via victim empowerment centres at local police stations. The youth centre where the programme is run has close co-operations with these centres. The programme is targeted at young adults and excludes schoolchildren. The women recruited to the programme were approached by the programme leaders and invited to voluntarily participate in the research. These women were divided between an intervention and a control group. The data collection ran independently of the programme.

The women in the control group received assistance to find a job or joined a computer workshop between the first and the second assessment time but did not receive any specific form of therapeutic intervention. They were provided an opportunity to join the therapeutic group at a later stage. 
The women in the intervention group were assigned to one of four programme runs of the women empowerment programme. These were implemented between 7 March 2016 and 29 April 2016.

A total of 103 women from both groups participated in an initial assessment, while 70 women participated in a follow-up assessment at the post-intervention time. Attrition was due to women finding jobs or leaving the region for some time. However, these factors were equally reflected in both intervention and control groups.

The analysis in this study is based on the 70 women who participated in both assessments. The intervention group comprised 39 persons who participated in the women empowerment programme and 31 formed the control group. The average age of all women in the study was $M=28.3, S D=8.4$; $34.29 \%$ were unemployed, $25.71 \%$ were working, $11.43 \%$ were studying, $27.14 \%$ were volunteers, and one participant was self-employed.

\section{Instruments}

All instruments used are multi-purpose screening instruments and do not appear on the list of classified psychological tests of the Health Professions Council of South Africa.

The PTSD Symptoms Scale-Interview for DSM-5 (PSS-I-5) was used to assess PTSD symptom severity (Foa \& Capaldi, 2013). The items correspond to the Diagnostic and Statistical Manual of Mental Disorders, Fifth Edition (DSM-5; American Psychiatric Association, 2013) symptom criteria for PTSD using the previous month as a point of reference. As the answers were rated on a 5-point scale ranging from 0 (not at all) to 4 (six or more times a week/severe), the maximum sum score is 80 and ranged from 0 to $47(M=10.56, S D=12.39)$ in this sample. The Cronbach's $\alpha$ coefficient in this sample was .91.

The Patient Health Questionnaire-9 (PHQ-9) is a self-report screening instrument consisting of nine items. It was used to screen the severity of depression symptoms (Kroenke, Spitzer, \& Williams, 2001) using the past 2 weeks as a point of reference on the first as well as the second assessment time after 12 weeks. The nine items refer to the $D S M-I V$ symptom criteria for major depression and a 10 th item assesses the everyday functionality of the women (Kroenke et al., 2001). As the answers are rated on a 4-point scale ranging from 0 (not at all) to 3 (nearly every day), the maximum sum score is 27 and ranged from 0 to $25(M=6.97, S D=5.52)$ in this sample. The cut-off points $5,10,15$, and 20 to describe a mild, moderate, moderately severe, and severe depression were aligned to other publications (Kroenke et al., 2001). The 10th item was analysed separately to assess the participants' level of functioning. A treatment recommendation was indicated if at least five statements applied to more than half of the days in the past 2 weeks or several days for suicidal ideations, they experienced their daily duties as difficult, and indicated depressive symptoms for more than half of the days in the past 2 weeks. The Cronbach's $\alpha$ coefficient in this sample was .84 .

Early adverse experiences were assessed using the Adverse Childhood Experiences International Questionnaire (ACE-IQ) (World Health Organization, 2016). It can be used with adults who answer all questions in reference to the first 18 years of their lives. It consists of 35 items that can be grouped into 13 categories reflected in a binary score. The ACE-IQ score indicates how many categories an individual has experienced and represents their overall adverse childhood experiences. The maximum score is 13 and ranged from 0 to 9 in this sample.

Participants were assessed between 1 and 3 weeks prior to the programme (baseline) and 12 weeks after completion of the programme (3-month follow-up).

\section{Procedure}

It was not possible to assign the women randomly to the two groups due to pre-enrollment in the programme for specific dates and other logistical factors. As such the researchers did not attempt 
to influence the enrolment procedure but maintained the process as it pre-existed. However, a randomisation check was used to ensure that the two groups did not differ significantly in potentially confounding variables and that a valid control group had been identified (Godin, Sheeran, Conner, $\&$ Germain, 2008). The mean values of relevant demographic variables and clinical scores were compared using Wilcoxon-Whitney $U$ tests. None of the Wilcoxon-Whitney $U$ tests comparing the mean value of the age, ACE score, PHQ-9 score, and PTSD sum score for the intervention and the control group reached significance (all $p>.05$ ). Such processes and measures have been similarly used in other clinical studies (Godin et al., 2008; Sheeran \& Orbell, 2000).

Two assessment procedures were conducted. The initial processes took place between 29 February and 29 April and the women were invited to the follow-up assessments after approximately 12 weeks between 1 June and 22 July.

The assessors consisted of volunteers and researchers. They were trained simultaneously and regular supervision was provided to ensure standardisation of the processes. The language of communication was English and one assessment was conducted in Xhosa with the help of a local and trained interpreter. The length of each assessment process varied between 45 and $120 \mathrm{~min}$.

Of the four women empowerment programmes, two programmes ran over 5 consecutive days and two over 4 days. Three of the programmes comprised 10 participants, while one had 9 participants. Each standardised programme consisted of 12 sessions as specified in the programme manual.

While the programme has a set structure, much time in the sessions is spent on sharing stories, self-reflection, and facilitated group narrations. The structure consists of an introduction, a closure, and 10 sessions outlined in the manual of the programme. Sessions comprise topics on selfenhancement, dealing with self-esteem, forgiveness, victim-mentality, and how to say 'no'. Other topics focus on domestic violence and physical, sexual, emotional, and economic abuse. Other sessions cover value clarification dealing with respect, courage, citizenship, fairness, care, diligence, and integrity. In a session on building relationships, the women examine their various relationships. Two sessions focus on the notion of PTG (Tedeschi \& Calhoun, 1996). This aims to prepare the women to manage negative emotions and stress. They fill out a survey assessing their risk of burnout, as well as their locus of control and learn about causal factors of these concepts. The manual also contains some relaxation techniques. They discuss the concept of beauty and subtle influential factors on feelings about physical appearance. In one session, the concept of grief, loss, trauma, and the five stages of bereavement are defined.

\section{Ethical considerations}

All women signed a consent form after reading through the information sheet that outlined the purpose and the implementation of the study, the regulations concerning confidentiality, data storage, and the possibility to receive counselling if required at any stage. The women received biscuits and cold drinks as refreshments after the assessment. No financial compensation was provided. The assessor noted the answers of the women on an answer sheet that was anonymised using specific codes. The ethical clearance was issued from the University of Pretoria in South Africa and the University of Konstanz in Germany.

\section{Data analysis}

The free software R (R Core Team, 2015) was used to analyse the data. Descriptive statistics were used to assess variables for the first assessment time. The age, the PHQ-9, and the PSS-I-5 score using Mann-Whitney U tests and the effect size $r=Z / \sqrt{n}$ were used to assess any significant differences between the women in the intervention and the control groups at the first assessment time. The PHQ-9 scores and the PSS-I-5 scores did not reflect a normal distribution. The mean 
Table I. Values for the intervention and the control groups at the first assessment time.

\begin{tabular}{|c|c|c|c|c|c|c|c|c|c|c|}
\hline \multirow[t]{2}{*}{ Variable } & \multicolumn{3}{|c|}{ Intervention group } & \multicolumn{3}{|c|}{ Control group } & \multicolumn{4}{|c|}{ Group effect } \\
\hline & $n$ & $M$ & $S D$ & $n$ & $M$ & $S D$ & $U$ & $Z$ & $p$ & $r$ \\
\hline Age & 37 & 27.77 & 8.29 & 37 & 29.06 & 8.58 & 681.5 & 0.91 & .365 & .11 \\
\hline PHQ-9 score & 37 & 6.46 & 5.82 & 37 & 7.39 & 5.24 & 689.5 & -1.01 & .316 & -.11 \\
\hline PSS-I-5 score & 31 & 7.97 & 11.69 & 26 & 9.48 & 10.86 & 547.5 & -0.76 & .454 & -.09 \\
\hline
\end{tabular}

PHQ-9: Patient Health Questionnaire-9; PSS-I-5: PTSD Symptoms Scale-Interview for DSM-5.

value and the standard deviation for both assessment times were assessed to show the comparative changes for the women in the intervention and control groups using Wilcoxon signed-rank tests and the effect size $r=Z / \sqrt{n}$. The difference of each score between the first and the second assessments was calculated and compared between the intervention and control groups using the Mann-Whitney $U$ tests and the effect size $r=Z / \sqrt{n}$. The level of significance was set to $p<.05$.

\section{Results}

At the first assessment time, 57.14\% (40) of the 70 women in the study indicated that it was not at all difficult for them to do their work, take care of the house, and get along with other people. A total of $22.86 \%$ (16) said it was somewhat difficult, $14.29 \%$ (10) said it was very difficult, and $5.71 \%$ (4) said it was extremely difficult. A total of $47.14 \%$ (33) had no signs of depression, 27.14\% (19) had mild signs, $15.71 \%$ (11) had moderate signs, $7.14 \%$ (5) had moderately severe signs, and $2.86 \%(2)$ had severe signs. In total, $12.86 \%$ (9) received a treatment recommendation for depression and $7.14 \%$ (5) met the criteria to be diagnosed with PTSD.

\section{Randomisation check}

As displayed in Table 1, Wilcoxon signed-rank tests revealed no significant difference between the two groups at baseline. In addition, a chi-square test showed no significant difference of severity depression between the groups, $\chi^{2}=4.45, p=.35$.

\section{Comparisons between the first and second assessment times}

Figures 1 to 3 give a graphical overview of the intervention effects in the three main outcome variables: PHQ-9, the functionality of the women in everyday life, and PSS-I-5.

\section{PHQ-9}

A one-way repeated measures analysis of variance (ANOVA) was conducted to compare the effect of time on the PHQ-9 scores of the women on the first and the second assessment time. There was a significant effect of time on the PHQ-9 scores of the women $(M=8.87, S D=5.55$ at the first; and $M=4.71, S D=4.50$ at the second assessment time), Wilks' Lambda $=.897, F(1,68)=7.83, p=.007$. Thus, depression symptoms declined in both groups between the two points of assessment.

There was however no significant interaction between the time and the group of the women, Wilks' Lambda $=.967, F(1,68)=2.308, p=.133$. On a group level, the mean PHQ-9 score for all women in the intervention group dropped from $M=6.46, S D=5.82$, before to $M=5.41, S D=5.14$, after the intervention (Figure 1). 


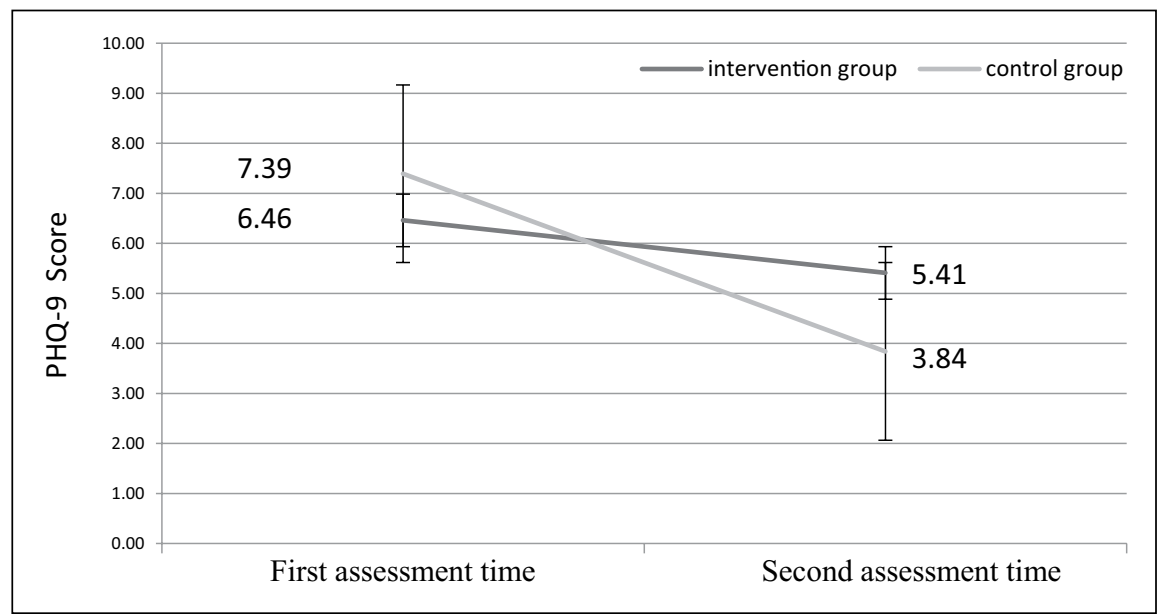

Figure I. Changes of values for the core measures between the first and second assessment times for the intervention and the control groups.

Assessed scores at the first and second assessment times as well as the comparison between the two assessment times for the intervention and the control groups. Error bars represent standard errors. Points are offset horizontally so that error bars are visible.

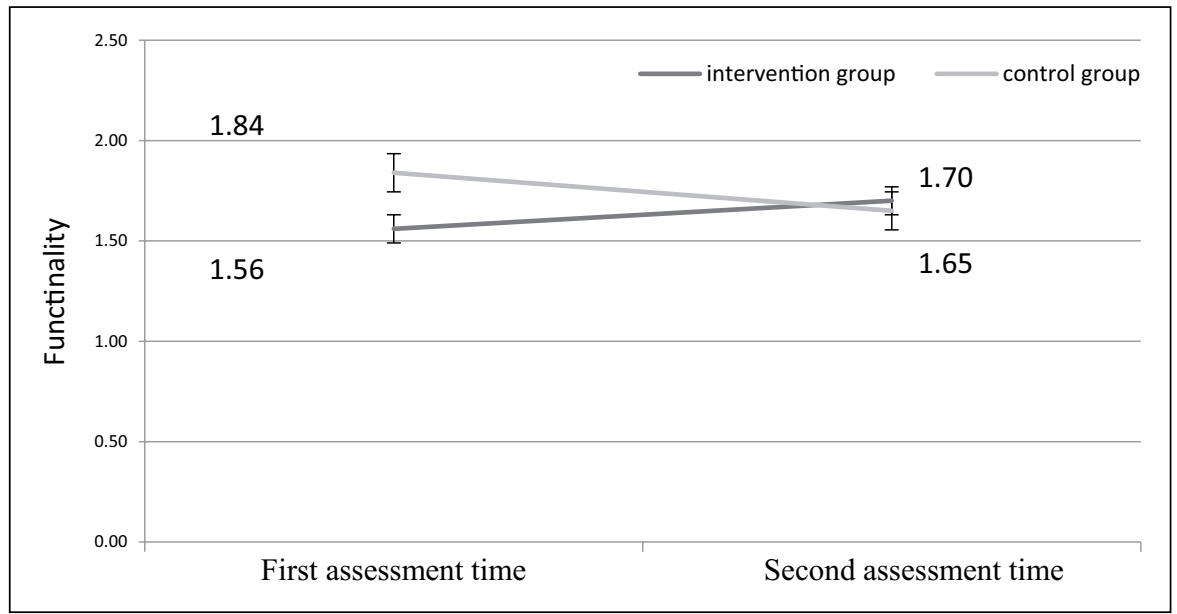

Figure 2. Changes of scores indicating the functionality of the women in everyday life.

Assessed scores at the first and second assessment times as well as the comparison between the two assessment times for the intervention and the control groups. Error bars represent standard errors. Points are offset horizontally so that error bars are visible.

A Wilcoxon signed-rank test however showed no significant effect of time ( $W=676, Z=-0.73$, $p=.399, r=-.12$ ). The same score dropped from $M=7.39, S D=5.24$, on the first to $M=3.84$, $S D=3.43$, on the second assessment time for women in the control group and the Wilcoxon signedrank test showed a significant time effect $(W=284.5, Z=-3.07, p<.001, r=-.55)$. The difference between the PHQ-9 score at the first and the second assessment times was $M=-1.05, S D=7.53$, for all women in the intervention and $M=-3.55, S D=5.82$, for all women in the control group. 


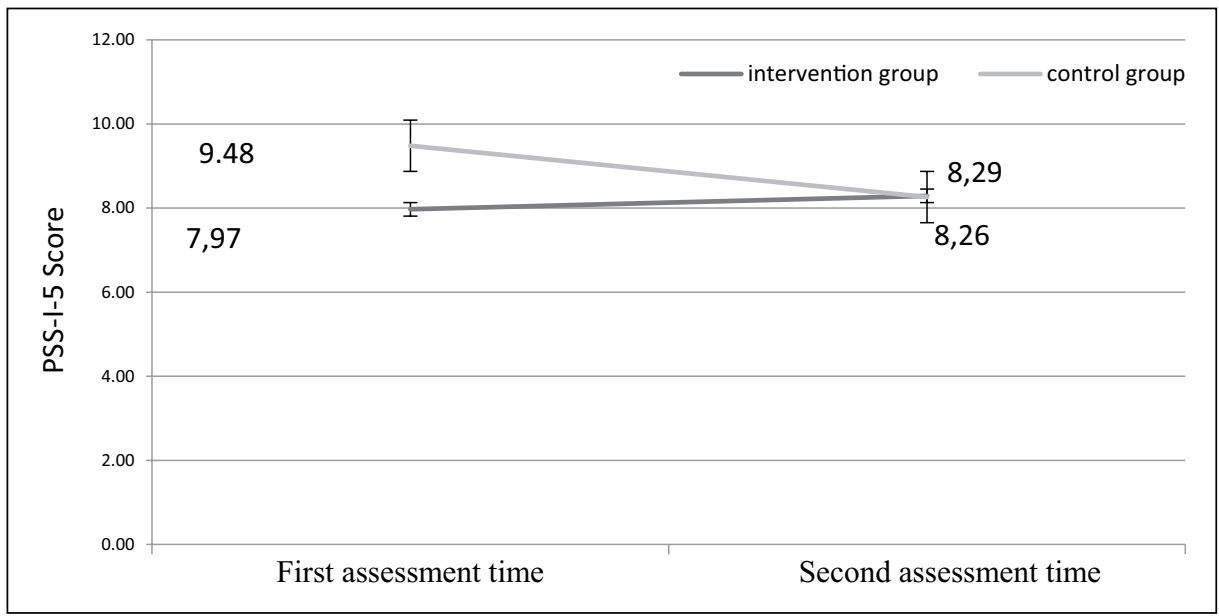

Figure 3. Changes of PSS-I-5 score.

Assessed scores at the first and second assessment times as well as the comparison between the two assessment times for the intervention and the control groups. Error bars represent standard errors. Points are offset horizontally so that error bars are visible.

The Mann-Whitney $U$ test showed a significant trend $(p<.10)$ between the two groups $(U=452.5, Z=-1.80, p=.073, r=-.22)$, indicating that the intervention group tended to report more depression symptoms than the control group following intervention.

\section{Changes in the level of functioning}

The one-way repeated measures ANOVA comparing the effect of time on the functionality of the women showed no significant effect of time on the functionality score, Wilks' Lambda $=.999$, $F(1,68)=.045, p=.832$, and no significant interaction between the time and the group of the women, Wilks' Lambda $=.984, F(1,68)=1.093, p=.299$.

The mean value of the Likert-type scale indicating the functionality of the women in everyday life was $M=1.56, S D=0.85$, before compared to $M=1.7, S D=0.83$, after the intervention for the women in the intervention group.

A Wilcoxon signed-rank test showed no significant time effect ( $W=847, Z=0.88, p=.406$, $r=.14$ ). The same value was $M=1.84, S D=1.00$, at the first, compared to $M=1.65, S D=.88$, at the second assessment time for the women in the control group. A Wilcoxon signed-rank test showed no significant time effect either $(W=433.5, Z=-0.85, p=.420, r=-.15)$.

\section{PSS-I-5}

The one-way repeated measures ANOVA comparing the effect of time on the functionality of the women showed no significant effect of time on the PSS-I-5 value, Wilks' Lambda $=.998$, $F(1,55)=.121, p=.729$, and no significant interaction between the time and the group of the women, Wilks' Lambda $=.987, F(1,55)=.724, p=.398$.

The mean PSS-I-5 value of all women in the intervention group was $M=7.97, S D=11.69$, before compared to $M=8.29, S D=11.28$, after the intervention.

A Wilcoxon signed-rank test showed no significant time effect $(W=595, Z=0.40, p=.631$, $r=.08$ ). It decreased from $M=9.48, S D=10.86$, on the first compared to $M=8.26, S D=8.99$, on the 
second assessment time for the women in the control group and a Wilcoxon signed-rank test showed that there is no significant time effect $(W=353, Z=-0.86, p=.397, r=-.16)$. The difference between the score at the first and the second assessment timew was $M=0.74, S D=12.72$, in the intervention group and $M=-1.77, S D=8.75$, in the control group.

The Mann-Whitney $U$ test showed no significant effect $(U=355.5, Z=-0.76, p=.451, r=-.1)$.

\section{Discussion}

Addressing PTSD and related symptoms are important stated objectives of the programme as it exists. However, relatively few women entering the programme after referral from the police stations and the victim empowerment agencies, measured symptoms that constitute a PTSD or depression, as a related symptom of PTSD. This suggests that the programme's strong focus on traumatic experiences may not be the most appropriate focus for this target group.

The results of the randomisation check showed that the intervention and control groups were comparable concerning the main outcome variables that were assessed in this study. However, only the women in the control group measured a significant reduction in the depressive symptoms while these variables did not change significantly for women in the intervention group. Participation in the programme seemed to inhibit an improvement on this outcome variable, as indicated by the significant trend.

The number of women in this study who measured depressive or PTSD symptoms on intake was relatively low compared to other studies in similar set-ups (Dinan et al., 2004). This suggests that the participation of all women in all the modules of the programme as it exists may not be suitable. However, the sample size did not allow subsequent analyses with sub-samples within the groups.

One option to increase the impact of the intervention may be to improve the selection process of participants prior to entering the programme. This could be achieved through the use of a twostep assessment consisting of a short screening and a reliable assessment to identify only those women who show a sufficient burden of psychopathology related to trauma (Saltzman et al., 2001). Another option may be to adjust the content of the programme to address more specific needs of women in a specific community. A trauma- and depression focussed element could remain as an 'add on' for those women showing clinically relevant mental health symptoms.

It is recommended that the programme under evaluation be adjusted to be more 'sensitive and responsive to the social, political, linguistic, economic, and spiritual realities' of the target group (Kirmayer, 2012, p. 158).

Results suggest that an intervention that aims to address women's well-being in a community that is shaped by a lack of resources could benefit from moving beyond the individual level to create a health-enabling environment. This study provides empirical data that may serve as a theoretical foundation for the development of a new composition of elements for the intervention. Specific questionnaires could capture the specific challenges in the participating women's environment. This suggests a participatory approach to designing women's empowerment programmes. Close collaborations between practitioners and researchers may be used to develop interventions that are tailored to identify needs of women in a specific target group.

The results of this study suggest that an assignment of participants to an intervention that is not specifically tailored to their needs may not have beneficial effects in the main outcome variables.

Limitations to this study include the time interval of 3 months post-intervention which may not measure the long-term treatment effect. However, previous research on the effectiveness of trauma therapy clearly demonstrates that the time interval chosen in this study is sufficient to uncover treatment effects or at least significant trends (Schaal, Elbert, \& Neuner, 2009). 


\section{Conclusion}

The research offers important findings on the limitations of community-based programmes that are not specifically tailored to the target group's needs and adds knowledge to the question of what works in the field of community psychology. More importantly, however, the study shows that community programmes that are based on face validity of different problems are not necessarily helpful and highlights the need for rigorous evaluations of such interventions.

\section{Funding}

The author(s) received no financial support for the research, authorship, and/or publication of this article.

\section{ORCID iD}

Linda E Blokland (iD) https://orcid.org/0000-0003-2840-4720

\section{References}

American Psychiatric Association (2013). Diagnostic and Statistical Manual of Mental Disorders (5th ed.). Arlington, VA: American Psychiatric Publishing.

Berkowitz, S. J., Stover, C. S., \& Marans, S. R. (2011). The child and family traumatic stress intervention: Secondary prevention for youth at risk of developing PTSD. Journal of Child Psychology and Psychiatry, 52, 676-685.

Bisson, J. I., Brayne, M., Ochberg, F. M., \& Everly, G. S. (2007). Early psychosocial intervention following traumatic events. American Journal of Psychiatry, 164, 1016-1019.

Bonanno, G. A. (2004). Loss, trauma, and human resilience: Have we underestimated the human capacity to thrive after extremely aversive events? American Psychologist, 59, 20-28. doi:10.1037/0003066X.59.1.20

Campbell, C., \& MacPhail, C. (2002). Peer education, gender and the development of critical consciousness: Participatory HIV prevention by South African youth. Social Science \& Medicine, 55, 331-345.

De Arellano, M. A., Deblinger, E., Cohen, J. A., Danielson, C. K., \& Mannarino, A. R. (2005). Community outreach programme for child victims of traumatic events: A community-based project for underserved populations. Behaviour Modification, 29, 130-155. doi:10.1177/0145445504270878

Dinan, B. A., McCall, G. J., \& Gibson, D. (2004). Community violence and PTSD in selected South African townships. Journal of Interpersonal Violence, 19, 727-742.

Fallot, R. D., \& Harris, M. (2002). The trauma recovery and empowerment model (TREM): Conceptual and practical issues in a group intervention for women. Community Mental Health Journal, 38, 475-485.

Foa, E. B., \& Capaldi, S. (2013). Manual for the administration and scoring of the PTSD Symptom ScaleInterview for DSM-5 (PSS-I-5). Retrieved from https://www.istss.org/ISTSS_Main/media/Documents/ PSSIManualPDF1.pdf

Godin, G., Sheeran, P., Conner, M., \& Germain, M. (2008). Asking questions changes behavior: Mere measurement effects on frequency of blood donation. Health Psychology, 27, 179-184.

Harvey, M. R. (2007). Towards an ecological understanding of resilience in trauma survivors: Implications for theory, research, and practice. Journal of Aggression, Maltreatment \& Trauma, 14, 9-32.

Havenaar, J. M., Geerlings, M. I., Vivian, L., \& Collinson, M. (2008). Common mental health problems in historically disadvantaged urban and rural communities in South Africa: Prevalence and risk factors. Social Psychiatry and Psychiatric Epidemiology, 43, 209-215.

Kerig, P. K., Fedorowicz, A. E., Brown, C. A., \& Warren, M. (2000). Assessment and intervention for PTSD in children exposed to violence. Journal of Aggression, Maltreatment \& Trauma, 3, 161-184. doi:10.1300/J146v03n01 11

Killen, T., Hien, D., Campbell, A., Brown, C., Hansen, C., Jiang, H., \& Nunes, E. (2008). Adverse events in an integrated trauma-focused intervention for women in community substance abuse treatment. Journal of Substance Abuse Treatment, 35, 304-311. 
Kirmayer, L. J. (2012). Rethinking cultural competence. Transcultural Psychiatry, 49, 149-164.

Kroenke, K., Spitzer, R.L., \& Williams, J.B. (2001). The PHQ-9: Validity of a brief depression severity measure. Journal of General Internal Medicine, 16, 606-613. doi: 10.1046/j.1525-1497.2001.016009606.x

Lombardo, T. W., \& Gray, M. J. (2005). Beyond exposure for posttraumatic stress disorder (PTSD) symptoms: Broad-spectrum PTSD treatment strategies. Behaviour Modification, 29, 3-9. doi:10. $1177 / 02012445504270853$

Murray, M., Nelson, G., Polan, B., Maticka-Tyndale, E., \& Ferris, L. (2004). Assumptions and values of community health psychology. Journal of Health Psychology, 9, 323-333. doi:10.1177/1359105304040897

Orsillo, S. M., \& Batten, S. V. (2005). Acceptance and commitment therapy in the treatment of posttraumatic stress disorder. Behaviour Modification, 29, 95-129. doi:10.1177/0145445504270876

Pillay, S. (2008). Crime, community and the governance of violence in post-apartheid South Africa. Politikon: South African Journal of Political Studies, 35, 141-158. doi:10.1080/02589340802366943

Pupavac, V. (2001). Therapeutic governance: Psycho-social intervention and trauma risk management. Disasters, 25, 358-372.

R Core Team. (2015). R: A language and environment for statistical computing. R Foundation for Statistical Computing, Vienna, Austria. Retrieved from https://www.R-project.org/

Saltzman, W. R., Pynoos, R. S., Layne, C. M., Steinberg, A. M., \& Aisenberg, E. (2001). Trauma- and grieffocused intervention for adolescents exposed to community violence: Results of a school-based screening and group treatment protocol. Group Dynamics: Theory, Research, and Practice, 5, 291-303.

Schaal, S., Elbert, T., \& Neuner, F. (2009). Narrative exposure therapy versus interpersonal psychotherapy. Psychotherapy and Psychosomatics, 78, 298-306.

Sheeran, P., \& Orbell, S. (2000). Using implementation intentions to increase attendance for cervical cancer screening. Health Psychology, 19, 283-289.

Shefer, T., Crawford, M., Strebel, A., Simbayi, L. C., Dwadwa-Henda, N., Cloete, A., \& Kalichman, S. C. (2008). Gender, power and resistance to change among two communities in the Western Cape, South Africa. Feminism \& Psychology, 18, 157-182.

Tedeschi, R. G., \& Calhoun, L. G. (1996). The posttraumatic growth inventory: Measuring the positive legacy of trauma. Journal of Traumatic Stress, 9, 455-471.

World Health Organization. (2016). Adverse Childhood Experiences International Questionnaire (ACE-IQ) Guidance for analysing $A C E-I Q[P D F]$. Retrieved from http://www.who.int/violence_injury_prevention/violence/activities/adverse_childhood_experiences/en/ 